\title{
Smart Materials Group at the National Institute of Applied Science Recent Data and Trends
}

\author{
Pierre. F. Gobin*, Yves Jayet, Phillipe Guy, Nathalie Godin, Michel Morin, \\ Catherine Gauthier, Xavier Kléber, Pascal Reynaud, \\ Joel Courbon and Jean Y. Cavaillé
}

Groupe d'Etude de Métallurgie Physique et de Physique des Matériaux, G.E.M.P.P.M, UMR CNRS 5510, Institut National des Sciences Appliquées de Lyon France, de LYON, Bât. Blaise Pascal, 7, avenue Jean Capelle, 69621 Villeurbanne Cedex - France

\begin{abstract}
During the last decade constant improvements have been made in materials and structures design and control. But now some performance objectives in particular in the field of the reliability cannot be achieved using classical technologies and require the use of the 'smart materials concept'. Periodical maintenance NDT based inspections are today of a general acceptance for almost all complex technological structures. Nevertheless the idea that the integrated and continuous sensing techniques can optimize the operating conditions is now in progress. Different aspects of this evolution towards the desirable continuous health monitoring are discussed in relation with the smart materials concept through this non exhaustive review of some realisations or experiences. In the domain of sensitive materials, passive techniques such as Barkhausen effect, thermoelectric power, electrical impedance monitoring, acoustic emission, and active piezoelectric implant based methods, are briefly presented. Moreover, taking into account the growing demand in the field of actuators and artificial muscles for robotic and biomimetic devices, shape memory alloys and electroactive polymers are in progress. Finally the 'self healing' concept will be presented in the case of one ceramicceramic composite.
\end{abstract}

(Received November 6, 2003; Accepted January 9, 2004)

Keywords: smart materials, multifunctional materials, health and damage monitoring, Barkhausen effect, thermoelectric power, acoustic emission, active piezoelectric implants, actuators, shape memory alloys, electroactive polymers, residual life time prediction, self healing

\section{Introduction}

The idea of 'smart' materials and structures evolved in the United States in early 1980's as the potentially viable solution to the problem posed by the next generation of -particularly military- aircraft. At the same time, the concept of 'intelligent' and even 'wise' materials was pioneered in Japan with the purpose of establishing a new area in materials science which would take into account the relationship between materials, the natural environment, and society new requirements -in the general frame of the sustainable development-. This concept has been at first proposed in a really 'biomimetic' frame in which a material would possess as a living system 'nerves' simulated by a network of sensors, 'muscles' or active parts and a 'brain' in order to coordinate the whole. This ambitious concept leads naturally to the multifunctionality for the structural materials and to the most difficult of the challenges: the self healing behaviour!

So, the "smartness" or 'intelligence' can be defined at three levels, each of them defining a field of research, technology development and potential applications:

- a material or a material-system is said to be "sensitive" when it behaves as a sensor or when it includes sensors providing information concerning the material itself or its environment.

- a material will be "adaptable" if it behaves itself as an actuator or if integrated actuators (or "active" materials) can modify its characteristics. Such a material or material-system will be adaptable only by the way of an externally determined action.

- The combination of these two above mentioned proper-

*Contact author, E-mail: pierre-francois.gobin@insa-lyon.fr ties results in an "adaptive" or "really smart" material which collects data related to the changes in its environment or in its own evolution or damage. It processes these collected data, and reacts through its 'actuators' action. However the techniques for appropriate response functions seem until now rarely addressed.

The question is now: how does work a sensor or an actuator necessary to develop sensitive, adaptive or smart materials (or materials systems )? In fact when a material is submitted to an optical, mechanical, electrical, thermal..., eventually biochemical prompting, it generates a similar or different effect. ${ }^{1)}$ Of course it is important for the designers of future systems to know the current level, the trends and the limits of the actuation or sensitive properties as well as the using conditions linked to the physico-chemical characteristics for each type of materials or materials-systems.

So it was in the late 1980's when the term 'smart' crept into European vernacular ${ }^{2)}$ that, we decided to launch in the * 'Groupe d'études de Métallurgie Physique et de Physique des Matériaux' a 'smart group' devoted to the active and sensitive structural materials. For that, we took in account our already well known skill in the field of the shape memory effect on copper and titanium based alloys ${ }^{3)}$ and the more recently developed piezoelectric implants technique ${ }^{4)}$ monitoring the composite materials evolutions. Effectively we recognized early, ${ }^{5)}$ the potential major role of composites in the field of the smart materials and systems because in this type of material 'smart functions' are created by the assembly of the components at the mesoscopic scale. Sensors and actuators can be incorporated into the components during the manufacturing process of the composite, for example in prepregs, woven fabrics, pultrusion or filament winding. 
Moreover, the idea that the integrated and permanent sensing techniques, can optimize the operating conditions is now in progress. In the first part of this paper, different aspects of this evolution towards the continuous health monitoring are discussed in relation with the smart materials concept, through a non exhaustive review of some realisations or experiences essentially focused in the field of sensitive materials.: polymers based composites and metallic alloys.

Furthermore, in order to propose real adaptive materials and or materials systems, active components have to be used $^{6)}$ and two examples are presented in the second part: shape memory alloys and electroactive polymers. Finally the last part of this text is devoted to the study of the chemical 'self-healing' behavior of one ceramic-ceramic composite.

\section{Sensitive Materials and Materials-systems}

The most natural way to obtain a "sensitive" material is to use the material itself or a part of the material-system (or composite) as sensor. Evidently that opportunity exists for metallic alloys and some composites, taking benefit of the relation between some physical phenomena and relevant features of the microstructure. Microstructure and mesostructure are known to play a fundamental role in properties of materials. Hence, characterizing microstructural and mesostructural features in a continuous or semi-continuous manner by a non destructive way is of a fundamental interest for the scientist who aims at a better understanding of mechanisms involved in thermo-mechanical processes as well as for the producer and the end-user who want to assess and monitor materials quality.

\subsection{Non classical non destructive techniques for mon- itoring structural conditions of metallic alloys}

In this domain our work is focused on two non classical non destructive techniques able to be used in continuous or quasi continuous microstructure monitoring of metallic components: the Barkhausen noise ${ }^{7)}$ (applicable only to ferromagnetic materials) and the thermo-electric power. ${ }^{8)}$ This interest is justified by the great sensitivity of these physical phenomena to the metallurgical condition in the predamaged state (solid solution foreign atoms, precipitates, dislocations...).

\subsubsection{Barkhausen effect}

In ferromagnetic materials a magnetic microstructure is made up of Weiss domains separated by Bloch walls, in interaction with the metallurgical microstructure of the material. When it is subjected to a varying applied magnetic field, this magnetic microstructure is reorganised through the collective motion of Bloch walls. However, the motion occurs in a jerky manner, due to the local interactions between domain walls and microstructural heterogeneities, thus inducing high frequency magnetic flux variations and leading to the so-called Barkhausen noise. Hence, each modification of the metallurgical microstructure (grain size, phase nature...) or obstacles to the wall motion (dislocations, precipitates, grain boundary singularities...) modifies the Barkhausen noise signature. For example, the pearlite response is different from the martensite one, due to a different magnetic microstructure. ${ }^{9}$ Furthermore, for a given phase, the creation of intragranular precipitates, which act as obstacles to domain wall motions, induces a more intense response.

Moreover, it should be mentioned that Barkhausen noise is also sensitive to applied or internal stresses, due to the magneto-elastic coupling, which can be also exploited to get information from the internal stress state of the material. An applied tensile stress increases the Barkhausen noise signal whereas a compressive one induces a decrease. Second order internal stresses due to plastic deformation can also modify the Barkhausen noise response. But in this case, two competitive mechanisms (second order internal stresses and generation of dislocations) are active and contribute to the total response. Hence, one has to separate the influence of each other if one wants to quantify internal stresses. ${ }^{10)}$

\subsubsection{Thermoelectric power}

Thermoelectricity concerns the direct generation of an e.m.f. by thermal means, i.e. by subjecting a conductor to a temperature gradient. The thermo-electric power $S$ is the quantity that characterizes the ability of this conductor to generate such an e.m.f. As well as the resistivity, the thermoelectric power is due to electron mechanisms.

Using the Seebeck effect, the thermo-electric power can be measured with two different types of experimental setup. In the first one, a temperature gradient is generated throughout the sample. It is realized by placing it at the top of two temperature regulated blocks (with a temperature difference $\Delta T$ ) made up with the reference conductor. By measuring the difference of potential $\Delta V$ between the junctions one can get the thermo-electric power of the sample $(S=\Delta V / \Delta T)$. The second one uses a small hot tip that ensures the contact with the sample. A thermal gradient is then generated locally below the contact area. The latter technique makes it possible to draw $2 D$ maps of $S$, even in industrial environment, when the hot tip is coupled with a X-Y table. The thermoelectric power is strongly influenced by various microstructural features of materials (composition, phases, defects...), but the main interest is certainly the sensitivity to the precipitation process. ${ }^{11)}$

\subsubsection{Complementarity of Barkhausen noise and ther- moelectric power}

Finally it has been shown ${ }^{7)}$ that Barkhausen noise and thermo-electric power measurements are both strongly influenced by the microstructure of materials and, in turn, then can be used as non-destructive techniques for characterising and monitoring microstructure evolutions in the predamaged state of metallic components. Nevertheless, their multiple sensitivity sometimes makes it difficult to deconvoluate all the information included in the measurements. Overcoming such a difficulty requires a good understanding of the phenomena and models relating physical mechanisms to measured quantities. ${ }^{10)}$

\subsection{Electro-mechanical coupling: towards the sensitive micro and nanocomposites}

The measurement of electrical resistance or impedance is among the most popular techniques able to monitor, eventually on line, the conditions of the materials during their usage. Obviously that opportunity exists in the well known case of Carbon Fibre Reinforced Polymers (CFRP) or 
hybrid glass-carbon FRP.

\subsubsection{Micro composites and nano fibres}

On the same way one of us (jyc) has developed in the frame of the CERMAV ${ }^{42)}$ a new type of micro-composite materials-systems with coupled electrical and mechanical properties $^{12-15)}$ These materials were obtained by casting of film forming latex containing electrically conducting whiskers. These whiskers are cellulose monocrystals with typical dimensions of $10 \mathrm{~nm}$ by $1 \mu \mathrm{m}$, coated by a very thin layer of a conducting polymer, polypyrrole (PPy) ${ }^{\mathrm{i}}$. Attention was paid to the dielectric behavior of these materials when they are submitted to a macroscopic deformation. When the conducting whiskers are randomly dispersed within the polymeric matrix, close (but above) the percolation threshold, the electrical conductivity is a complex number, with a real (or resistive) part due to the whisker network and an imaginary (mainly capacitive) part due to the dielectric interactions between the whiskers (accounting for the main branch conformation, dead branches and finite aggregates).

Two different behaviors can be exhibited. When the macroscopic tensile deformation is homogeneous, it leads to a decreases of the real conductivity due to random local breaks of the network, and at the same time to an increase of the capacitive conductivity. In that case, and roughly speaking, the macroscopic deformation, and in turn the network random local breaks act similarly to a decrease of the whisker fraction in the non deformed state which leads to the divergence of the dielectric constant at the percolation threshold. On the contrary, when the deformation is localized (such as necking), that leads to a macroscopic break of the network, leading in turn to a drop of both the real and imaginary parts of the conductivity.

Among the applications of such materials, their use as coating on various substrates playing the role of probe to detect local cracks ${ }^{16-18)}$ is promising.

\subsubsection{Nanocomposites and nanotubes}

At this moment, we start to work on nanocomposite materials consisting in a polymeric matrix with dispersed carbon nanotubes (CNT) in it ${ }^{19,20)}$ Here again, an interesting coupling between mechanical and electrical properties is expected. However, the processing step is still a challenge, as carbon nanotubes are very difficult to randomly disperse avoiding the formation of aggregates. Nevertheless, such a dispersion was achieved for $3 \% \mathrm{vol}$ within a matrix of a film forming latex, with $T_{\mathrm{g}}$ around of $0^{\circ} \mathrm{C}$. In terms of mechanical reinforcement, the stiffness of the nanocomposite with $3 \% \mathrm{vol}$ of well dispersed CNT is increased by a factor of about $10 \mathrm{in}$ the rubbery range (i.e. above $T_{\mathrm{g}}$ ). In fact, the percolation threshold is much lower, and the maximum conductivity effect is expected, as recalled above, for composition close to the threshold. For higher contents, only a slight effect of the strain on the conductivity is exhibited.

\section{3 'Sensitive' classical composites with piezoelectric sensors}

The life duration and the health condition of a materialsystem or composite structure is closely linked to the evolution of its matrix material during the life cycle (ageing), as well as to the degradation associated to the presence of localized defects such as matrix or fibre cracks or delami- nations. Piezoelectric ceramics can be used as 'passive' sensors able to sense the strain waves associated to the initiation of the damage and travelling into the materials ('acoustic emission') or as 'active' sensors able to generate pressure and Lamb waves interacting respectively with the micromechanical properties related to the physico-chemical conditions of the matrix and with the mesoscopic and macroscopic defects of the structure.

2.3.1 Passive piezoelectric sensors and acoustic emission

The first type of sensor is used in the classical acoustic emission (AE) technique developed over the last two decades as a non-destructive evaluation technique and as an useful tool for material research ${ }^{21,22)}$ In stressed materials, the strain-energy release due to microstructural changes results in stress-wave propagation. Therefore, this technique potentially allows not only to locate the source of the emission, but also to determine its nature. However the stress waves resulting from the microstructural changes depend on the propagation conditions such as the ultrasonic attenuation in a heterogeneous medium, and also on boundary surface interactions. So, the signal delivered by the sensor is a strongly modified representation of the original source. Nevertheless, it is realistic to consider that this signal contains some features representative of the source in such a manner that correlation exists between the damage mechanisms and the magnitude of the various AE parameters. Consequently, each signal can be considered as the acoustic signature of the different damage modes. As an example, this technique has been used to characterise damage evolution in different types of glass fibers-polyester composites that we expected to produce preferential damage mechanisms. ${ }^{23)}$ Two main types of signals were identified denoted A and B, coming from the two awaited damage mechanisms: matrix cracking (A) and fiber/matrix decohesions (B). The initiation, the growth of matrix cracks and their coalescence appear to create AE events having low amplitude, slowly rising and decaying. On the contrary, the interface debonding leads to signals with higher amplitude, quickly rising and decaying. But the difficulty to separate objectively the two types of signals within a large quantity of signals lead us to use neural networks based pattern recognition techniques. So, the combination of acoustic emission amplitude distributions and neural networks, in the form of a Kohonen selforganising map was successfully employed to discriminate signals originating from different damage types. At a macroscopic level, the cumulative plot of the events of each class versus time clearly indicates damage initiation and progression of each failure mechanism during tensile tests. This work can lead to perspectives like real-time detection of damage in complex GFR structures, by analysing the evolution of AE activity originating from the 'most dangerous' damage modes.

\subsubsection{Application to the residual Life time prediction}

Moreover it is interesting to note that this type of information can be used in order to propose a semi phenomenological approach for the residual life time prevision. Effectively beyond the health monitoring concept a real challenge concerning materials and structures is their residual life time prediction when subjected to wide variety of environmental and mechanical loading conditions that can 
initiate damage and lead to failure. The key issue in predicting lifetime of many different parts is evidently to use the results given by the continuous health monitoring. Indeed, damage at the smallest scales drives damage accumulation at larger length scales until some critical local damage state is attained that causes macroscopic failure. It appears necessary to characterize distributed localized than more global damage in order to understand the mechanisms of its initiation, evolution and criticality and so, to identify the relevant precursors of failure. Indeed acoustic emission which corresponds to the part of energy generated by the material during the damage processes is directly related to the damage mechanisms and so can give pertinent information about the damage initiation and development. The hypothesis that A.E. signals produced during damage creation and development contain relevant precursory information about damage that can be used in a failure prediction scheme seems reasonable. Failure mostly appears to be a critical point especially when it is not controlled by single events accumulation (nucleation and growth of microcracks) but rather by multiple events succession that leads to the catastrophic growth of a dominant crack. From this point of view failure can be seen as a co-operative phenomena. Indeed, in the case of complex systems, recent works using statistical physics approaches have shown that, in some cases, these statistical models successfully predict failure. ${ }^{24)}$ Some authors suggested that rupture of sufficiently heterogeneous media should exhibit some 'universal' behavior and in this field, recent works ${ }^{25)}$ have shown that $\mathrm{AE}$ has a great potentiality. It has been observed that the elastic energy released per unit time, on average, increases as a power law of the time-to-failure. The rupture was found to occur as the culmination of the progressive nucleation, growth and merging between microcracks. However, different tests carried out in the GEMPPM on polymer based composites have shown that the well known power law are only valid very close to the rupture. In fact, this result considerably limits the interest of a prediction approach. In order to increase the possibility of the predicting approach, different extrapolation laws have been proposed. Another important question that needs to be addressed concerns the influence of the size of the structure and of the type of loading on the law describing the evolution the AE signals. Among the main questions asked today, the precise role of the material microstructure has to be determined. The concept of universal behaviour leads to the idea that whatever the materials kind, its residual lifetime is only related to the energy released during its damage. In fact (i) this require that the damage should be spread inside the material , and (ii) that each event correspond to a brittle local behaviour Furthermore, it could be interesting to apply the predicting approach to the most relevant precursory AE signals, those governing the final rupture. For that purpose, the identification method described in the previous section could be used.

2.3.3 'Active' piezoelectric sensors:evolution of structural properties and detection of localized defects

The piezoelectric implant method differs from traditional techniques by the fact that the thin ceramic used is integrated into the composite structure during its elaboration. That enables to avoid the problem of the acoustic coupling reproducibility. The element dimensions can be determined in order to uncouple the frequency ranges of the thickness and radial vibration modes From the measurement of the electrical impedance of this embedded piezoelectric disc within the frequency range of its thickness mode one can recover some micromechanical parameters of the host structure in relation with the chemical properties of the matrix.

On the other hand, the radial vibrations are used to launch and detect Lamb-like waves, which are known to propagate over long distances. The guided waves also have the advantage to be multimodal. And the various propagating modes offer a specific sensitivity to the different kind of defects that a structure can host.

\subsubsection{Monitoring the curing and ageing conditions}

When such an element is inserted during processing in a composite, it is shown, by using a dimensionnal model based on the axial vibration, that its electrical impedance depends on the physical and geometrical parameters of the ceramic and on the viscoelastic characteristics (velocity and attenuation of plane waves) of the surrounding medium. It is therefore possible by solving the inverse problem by means of a non linear optimisation algorithm to extract ,by comparison between experimental and theoretical results, the attenuation and velocity values of this material, in relation with its structural conditions during curing and polymerisation $^{4)}$ Complementary experiments have shown that the evolution of these parameters are insensitive to the nature or the amount of fibres and represents only the polymerisation process of the resin.

After processing the piezoelectric implant remains inserted in the structure and its resonant properties will be an indicator of the structural evolution all along the lifetime of the composite structure. In particular water immersion may be an important factor of degradation which is known to decrease the mechanical properties. The evolution of the attenuation after immersion shows that the composite becomes more and more dispersive (attenuation is more frequency dependent). As the resin alone exhibit only a very slow and weak evolution, the variations of the composite viscoelastic properties are in relation with the degradation of the fibrematrix interfaces and the resulting attenuation increase may be used as a localized damage indicator. ${ }^{26)}$

This study has led to devising a computer controlled prototype enabling both the measurement of electric impedance of the piezoelectric implant, and its processing to extract in real time the acoustical parameters of the material to be characterized. ${ }^{27)}$ This device allows to monitor the acoustical properties during all the life cycle, polymerisation and ageing, and give in real time information about the structural conditions of the composite.

\subsubsection{Damage detection by use of guided waves gener- ated by piezoelectric discs}

The radial vibrations of the preceding PZT discs can also be exploited in order to generate low frequency guided waves able to propagate through plates and interact with significant damages such as holes, delaminations or microcracks. ${ }^{28,29)}$ The dimensions of the piezoelectric elements are determined in order to uncouple their thickness and radial vibration modes. The piezoelectric elements can then be arranged in a 
pitch-catch configuration in order to monitor the structure. In the low frequency range, a particular Lamb mode (either symmetrical or anti-symmetrical) can be selected by tuning the frequency of the applied voltage.

The knowledge of the various Lamb modes properties is essential to identify the components of the transmitted waveforms, to help data interpretation, to assess the different mode sensitivity to different kinds of defects, and also to locate defects. This requires the measurement of the material densities and stiffness tensors. The elastic moduli are measured using an ultrasonic characterisation device, developed in the laboratory.

To introduce realistic damages such as those observed during the service of real aeronautic structures such as accidental fall of a tool during maintenance, we chose lowspeed impacts, (i.e; velocities below 20-40 meters per second). To verify that the plate has effectively been damaged and also to highlight the actual size and shape of the internal induced damage, was verified through a classical C-scan procedure. This technique demonstrates that delaminations leading to a classical two-lobe pattern occur at the interfaces between plies having different orientations rather than between plies with the same orientation.

After each impact the sample is interrogated with propagating Lamb modes. This consists in exciting the sensor with a 5-cycles toneburst of adjustable frequency, in order to select a particular Lamb mode, and in recording the transmitted waveforms on the receiver, which is identical to the emitter. The $S_{0}$ mode was retained, as it appeared to give better results than the first anti-symmetrical Lamb mode $\mathrm{A}_{0}$ for the detection of "in-plane" flaws. Nevertheless, it is difficult to find a reliable discriminating parameter through the observation of the time signals.and easier to use spectral analysis and to introduce a new damage indicator: the damage index DI, DI represents the cumulated absolute value of the difference between a reference spectrum and the sample spectra calculated for each frequency point $i$. This quantity is normalized with respect to the absolute value of the reference spectrum. So $D I=0$ when the measured spectrum is identical to the reference one, and $D I=1$ when the damaged spectrum is nil. This occurs when no signal can propagate between the emitter and the receiver (the plate is totally broken).

With this new parameter the non damaging impacts give results very similar to the reference set when the damaging impacts are clearly discriminated by two very different values of the $D I$ index. ${ }^{30 \text { ) }}$

Moreover in order to simulate the interaction of guided Lamb waves with realistic damages, we used a finite elements approach. The delaminations are simulated by introducing very thin elements of negligible elastic properties with respect to those of the constitutive plies. In accordance with literature and with our experimental observations, delaminations have been introduced at the interfaces between cross plies only.

So the waveform calculated on a sample with a 'simulated' damage point shows a first echo which amplitude is lower than the first echo observed in the undamaged plate, followed by a second one of higher amplitude. The first echo can be ascribed to $S_{0}^{\prime}$ mode while the second one is du to the arrival of the converted $A_{0}^{\prime}$ mode.

By windowing the waveform, it is possible to isolate the $S_{0}^{\prime}$ contribution and to calculate the damage index $D I$, just as it has been done for the experimental measurement. This value is in really good agreement with the experimental one. in the case of a realistic simulation of the damage. ${ }^{31)}$

\section{Materials for Actuators}

The demand is growing in robotics and bioengineering for synthetic actuators with properties close to, those of biological muscles. Hence it is desirable to target performances which at least equal those of natural muscles but these objectives lead to goals difficult to realize together (high power/weigth ratio, fast response time, self control of strain, compliance....). ${ }^{32)}$

Two type of devices are now under consideration in our laboratory in this 'bioinspired' domain of 'smart materials: the shape memory alloys and more recently the electroactive polymers.

\subsection{Shape memory alloys}

The shape memory alloys (SMA) undergo by cooling martensitic transformations similar to those observed on a lot of metallic, ceramic and even partially crystalline polymer materials, and in particular on steels. But in the case of the commonly used SMA's (Cu-Zn-Al or Nickel Titanium) this first order transition is reversible with a small thermal hysteresis linked to a negligible volume variation. ${ }^{3)}$ Two important characteristics of martensitic transition are the absence of long-range diffusion and the appearance of a shape change. The shape memory alloys typically transform from a partially ordered cubic austenitic phase to a martensitic low temperature state. ${ }^{33)}$ This martensite easily deforms under stress by domain-wall displacements and when reheated goes back to the original shape of the high temperature structure: this is the one-way shape memory effect. The reversible relative strain can be as high as $8 \cdot 10^{-2}$ and the SMA's are good candidates in order to work as artificial muscles . It is also possible to obtain a reversible two way shape memory effect between high and low temperature shape by temperature cycling under applied stress or strain. This 'training' necessary in order to use such alloys as reversible actuators introduces in the austenite some bundles of dislocations homogeneously distributed which direct the growth of the martensite domains. ${ }^{34)}$ Moreover mechanical stresses can also cause phase change as well as the temperature, inducing the well known super elastic effect linked to the austenite-martensite transition.

An artificial muscle is a device able to supply mechanical work by transformation of an other form of energy. In the present case this calorific energy must be provided or released in order to change the temperature and to trigger of reverse and direct martensitic transformations during the socalled two way shape memory effect.

The most useful shape memory alloy the Ni-Ti alloy or 'nitinol' appears in 1963 and during the late 60's and early 70 's researchers started to develop applications in many fields from technical to medical domains. ${ }^{35}$ )

Nevertheless such actuators have to be monitored in order 
to adjust the strain to the desired effect and the simplest solution is to use the actuator itself as sensor. Effectively when a shape memory alloy (SMA) is thermally cycled with a constant load, changes in the strain $(\varepsilon)$ and the relative electrical resistivity $\left(\Delta \rho / \rho_{0}\right)$ occur in the material. If changes in strain and in electrical resistivity are measured simultaneously during this cycling, it is possible to build up curves of coupled strain-resistivity measurements. When a linear correlation in addition with a very weak hysteresis is observed in a shape memory alloy, this alloy can be used as sensor-actuator, in robotic devices. Actually, the control position quality of the actuator depends on the linearity and the hysteresis of the strain-resistivity curve for repeated cycles. Indeed, if the position of the actuator (strain) is followed through the resistivity, when some hysteresis is present, the true position detected will depend on the sign of the temperature change. The Ti-45.0Ni-5.0Cu at $\%$ alloy is a good candidate as sensor-actuator because of its one step transformation and the occurrence of a a fairly linear strainresistivity behaviour almost free of hysteresis after heat treatment at $385^{\circ} \mathrm{C}$ of a cold worked sample. ${ }^{36,37)}$

This important result where we use again coupled electrical and mechanical properties is paving the way towards really 'smart' devices in which a programmed strain would be easily controlled thanks to the reversible parallel evolving of the resistivity. ${ }^{38)}$

\subsection{Electroactive polymers: Actuators based on inter- penetrated polymer networks and electronic con- ducting polymer}

This study is performed in the general frame of the development of polymer based actuators, in collaboration with the Laboratoire de Physico-chimie des Polymères et des Interfaces from Université de Cergy-Pontoise. The investigated devices are based on the association, at the scale of the tenth of micron, between an electronic conducting polymer (polyethylenedioxythiophene or PEDOT) and a solid polymer electrolyte based on poly(ethylene oxide)/ polycarbonate $(\mathrm{PEO} / \mathrm{PC})$ interpenetrated polymer network (IPNs).

The devices are elaborated in two main steps. First, the solid polymer electrolyte (IPN) is synthesized, then the gradual dispersion of the electronic conducting polymer (PEDOT) on each side of the specimen is realized. (PEO/PC) IPNs were obtained by a two step in-situ radical polymerization of poly(ethylene glycol) dimethacrylate (PEGDM) and diethyleneglycol bis(allyl carbonate) (CR39). It is essential to tune as finely as possible the synthesis conditions of any IPN. In particular, the adjustment of the respective polymerization kinetics of the two networks (through temperature, initiator nature...) is a prerequisite to the structure control of the resulting IPNs.

Following the IPNs synthesis step, variable amounts of EDOT monomer are introduced and polymerized within the (PEO/PC) matrix. This leads to the formation of a gradient of PEDOT on each external layer of the device. The conductivity at the surface is found very similar to that of PEDOT obtained by bulk polymerization. Compared to conventional 'three layers' devices, the main advantage of this IPN based system is the lack of adhesion problem and the protection of the conducting polymer that may improve the durability of the device. At the moment, actuators are tested from these samples. After immersion in a $\mathrm{LiClO}_{4}$ solution, high deflection angles from $45^{\circ}$ to $90^{\circ}$ have been obtained under moderate applied potential from 0.5 to $2 \mathrm{~V}$. The deflection is reversible upon several cycles. The current study concerns the optimization of the lifetime of the device. ${ }^{39)}$

\section{The Self Healing Behaviour}

Effectively, beyond the continuous health monitoring, the ultimate goal for a smart material is to present a quasi biomimetic behaviour in order to ensure its survival. Examples are rare and till now only feasible by chemical way, but it appears possible to realise self-healing $\mathrm{SiC} / \mathrm{SiC}$ type composites. Ceramic matrix composites reinforced with long fibers are potential candidates for usage particularly in aerospace industry under severe conditions of temperature and environment. The SiC-SiC composites (Cerasep A410 ${ }^{\mathrm{TM}}$ produced by SNECMA for example) in which a SiC matrix is reinforcer by $\mathrm{SiC}$ fibers exhibit some favourable characteristics such as high mechanical properties and a reasonable resistance to high temperatures in spite of a certain sensitivity to interfacial degradation by oxidation at temperatures above $450^{\circ} \mathrm{C}$.

A complete experimental work has been realized on such a composite observed during cyclic fatigue in air at $600^{\circ} \mathrm{C}$ and $1200^{\circ} \mathrm{C}$ in order to study the damage accumulation resulting from mechanical fatigue and oxidation in relation with the fatigue lifetime. ${ }^{40)}$ This work was done in the frame of one academic-industrial partnership supported by the CNRS 'Modelling of behaviour and durability of ceramic matrix composites'. Material concept was based on the use of HiNicalon fibres, of a new self healing technology for the matrix and of a multilayer woven reinforcement to reduce delamination sensitivity during the manufacturing process.

The critical property of classical SiC-SiC composites is the poor oxidation resistance of pyrocarbone interphase, and in order to be opposed to this weakness, $\mathrm{SiC}-\mathrm{Si}$-B-C composites with a multilayer matrix, forming sealant glasses over a wide range of temperature $\left(450^{\circ} \mathrm{C}\right.$ to $\left.>1200^{\circ} \mathrm{C}\right)$ have been studied. Such multi layer matrix contain phases causing healing of the cracks and preventing oxygen to reach the pyrocarbon interphases.

The comparison of fatigue behaviour at $600^{\circ} \mathrm{C}$ and $200^{\circ} \mathrm{C}$ has highlighted two different damage mechanisms. Whereas high temperature fatigue $\left(1200^{\circ} \mathrm{C}\right)$ favours increasing inelastic strain and decrease of the macroscopic Young's modulus, at $600^{\circ} \mathrm{C}$ these parameters remain constant until the rupture. Microstuctural observations confirm the sudden breaking after fatigue at $600^{\circ} \mathrm{C}$ with a low pull-out length. On the contrary fracture surfaces of $1200^{\circ} \mathrm{C}$ tests show large debonding length. In both cases, the crack deflection in mode II is observed between the different layers of the matrix. Finally long fatigue life times in air at these temperature $\left(>1000 \mathrm{~h}\right.$ at $600^{\circ} \mathrm{C}$ under $\left.0 /+150 \mathrm{MPa}\right)$ attest the considerable improvement brought by this new multilayer matrix, as well as its protective role in the oxidation process of the fiber matrix interfaces. Just now new tests are in progress in order to precisely understand and possibly to improve this 
protecting role.

\section{Conclusions}

During the last decades constant improvements have been made on materials, manufacturing processes, structures design and control. Nevertheless, in parallel, the performances objectives have improved and now, it seems that some objectives cannot be achieved by the classical technologies. It is the reason why the smart materials based continuous health monitoring is emerging. ${ }^{41)}$ In particular in the domain of fibres reinforced composites in which the smart concept is relatively easy to develop, knowledge of operational conditions and information on the state of the structure by parameters measurements as strain, temperature moisture, or event detection such as impacts, delaminations, can lead to cost and cycle reduction. This improvement will be significant during manufacturing and control in operation and maintenance, as well as in improvement of safety, evidently by the opportunity to develop a warning before a fatal evolution of the structure and on a more prospective manner by the on line prediction of the residual lifetime. We are extending now this type of approach to some metallic alloys in the predamaged state and eventually in preliminary stages of the damage. At one other scale similar concepts are in progress in the promising field of micro and nanocomposites. In parallel, taking into account the growing demand in microrobotics and bioengineering for synthetic and musclelike actuators we develop from a long time our knowledge on shape memory alloys and more recently on electroactive polymers. Shape memory alloys based devices are becoming really 'smart' and one of our goal in electroactive polymers is to realize 'dry' actuators. Finally in spite of the illustrative examples concerning the slowing down of the damage growth and the self healing effect of one ceramic-ceramic composite or other material-systems a lot of improvements are necessary before a lot of smart materials-systems can properly fulfil all these requirements.

\section{REFERENCES}

1) B. Sillon, J. C. Dubois, P. F. Gobin, M. Pineri, M. Sixou and M. Vert: Cahiers de synthèse du CNRS en Science et Génie des Matériaux (1997) pp 65-106.

2) G. Beck and P. F Gobin: Journal of the Society of non traditional technology 2-3 (1992) 27-54.

3) G. Guénin, M. Morin, P. F. Gobin, W. Dejonghe and L. Delaey: Scr. Meter. 11 (1977) 1071-1075.

4) I. Perissin and Y. Jayet: 2nd International Conference on Intelligent Materials, Williamsburg, Virginia, June, 5-8 (1994) pp. 976-985.

5) P. F. Gobin, G. Guenin, M. Morin, M. Salvia and J. Tatibouet: J. of Intelligent Mat. Syst. and Strutures 7 (1996) 353-357.

6) P. F. Gobin, M. Salvia, L. David and M. Morin: Complex Adaptive Structures- Hutchinson Island USA, SPIE Vol. 4512 (2001) pp 84-92.

7) C. Gatelier-Rothea, J. Chicois, R. Fougères and P. Fleischmann: Acta Mater. 46 (1998) 4873.

8) R. Borrelly and J. L. Bouvier-Volaille: Traitements thermiques 221 (1988) 43.

9) O. Saquet, J. Chicois and A. Vincent: Mater. Sci. Eng. A269 (1999) 73.

10) X. Kleber, Y. Brechet and A. Vincent: Matériaux et Techniques, $\mathrm{N}^{\circ} \mathbf{9}$ 10 (2000) 39.
11) J. M. Leborgne: 'PHD Thesis INSA de Lyon (1996).

12) L. Flandin, G. Bidan, Y. Brechet and J. Y. Cavaillé: Polymer Composites, 21 (2000) 165-74.

13) L. Flandin, Y. Brechet, R. Dendievel and J. Y Cavaillé: J. of Mater. Sci. 34 (1999) 1753-59.

14) L. Flandin, M. Verdier, B. Boutherin, Y. Brechet and J. Y. Cavaillé: Journal of Polymer Science: Part B 37:8 (1999) 805-814.

15) L. Flandin, Y. Brechet, G. Canova and J. Y. Cavaillé, Modelling Simul Mat. Sci. Eng. 7 (1999) 865-74.

16) J. Y. Cavaillé and L. Flandin: 'Patent: 'Electrically Conductive Cellulosic Microfibrils and Matrices Reinforced Therewith' European Patent EP97400022 (BE, DE, FR, GB, IT, NL) January 1997. Extension to USA, to Japan, to China and to Korea, Attorney's Docket n 006050421, February (1997).

17) L. Flandin, Y. Bréchet and J. Y. Cavaillé: Compos. Sci. Tech. 61 (2001) 895-901.

18) L. Flandin, B. Bréchet and J. Y. Cavaillé: Proceedings of the 2nd Japan France Seminar on Intelligent Materials and Structures, ISBN 284512-1, (1999) 109-112.

19) F. Dalmas, L. Chazeau, C. Gauthier, J. Y. Cavaillé, R. Dendievel and L. Forro: Fourth Annual Meeting of the Group of Research on Carbon Nanotubes, Lyon, October (2003).

20) F. Dalmas: PhD Thesis, in preparation.

21) D. Rouby and P. Fleischmann: Phys. Status Solidi (A) (1978) 439.

22) D. Rouby, P. Fleishmann and P. F. Gobin: Revue du CETHEDEC $4{ }^{\text {ème }}$ trimestre (1980) p. 205.

23) S. Huguet, N. Godin, R. Gaertner, L. Salmon and D. Villard: Compos. Sci. Tech. 62 (2002) 1433-1444.

24) D. Sornette and C. Vanneste: Phys. Rev. Lett. 68 (1992) 612.

25) A. Guarino, A. Garcimartín and G. Ciliberto: European Phys. Journ. B, 6 (1999) 13-24.

26) Y. Jayet, N. Saint-Pierre, J. Tatibouet and D. Zellouf: Ultrasonics 34 (1996) 397-400.

27) Y. Jayet, J. C. Baboux and P. F. Gobin: Demonstrator workshop presentation, 4th ESSM and 2nd MIMR Conference, Harrogate (IOP publishing) (1998) pp. 797-799.

28) T. Monnier, Y. Jayet, P. Guy and J. C Baboux: J. Smart Mater. Struct. 9 , n'3 (2000) 267-272.

29) T. Monnier, Y. Jayet, P. Guy and J. C. Baboux: QNDE, Montreal 99, Review of Progress in QNDE, eds. D. O. Thompson and D. E. Chimenti, (Plenum, New York, AIP-Conference-Proceedings) 19B (2000) 1269-1276.

30) P. Guy, T. Monnier, J. C. Baboux and M. Salvia: J. Adv. Sci. 12, n³, (2000) 268-274.

31) P. Guy, Y Jayet and L Goujon: Smart Materials and Structures, San Diego (2003).

32) P. F Gobin, M. Salvia and M. Morin: 1st World congress on biomimetics and artificial muscles, Edt M. Shahimpoor, (Albuquerque, December 2002).

33) A. Planes, L. Manosa, E. Vives, J. Rodriguez-Carjaval, M. Morin, G. Guénin and J. Macqueron: J. Phys. Cond. Matter. 4 (1992) 553-559.

34) D. Rios-Jara: M. Morin, C. Esnouf and G. Guénin: Scr. Meter. 19 (1985) 441.

35) Y. Furuya: Proc. Intern. Symp. on Microsystems, Intelligent materials and Robots, Sendai, (1995) pp 313-318.

36) E. López-Cuéllar, G. Guénin and M. Morin: European Symposium on Martensitic Transformation and Shape Memory ESOMAT (2003).

37) E. López Cuéllar, C. J. De Araujo, G. Guénin and M. Morin: Ingenierías, Abril-Junio (2003), VI, No. 19, 31-38.

38) E. Lopez-Cuellar: PhD Thesis, INSA de Lyon, France, (2002).

39) C. Plesse, F. Vidal, D. Tessye, C. Chevrot and C. Gauthier: to be published.

40) O. Penas, P. Reynaud, D. Rouby and G. Fantozzi: Euro-Tecmat Congress Proceedings, Bucarest September (2000).

41) P. F. Gobin, M. Salvia, J. C. Baboux, N. Godin and Y. Jayet: 1st European Workshop on Structural Health Monitoring 2002, (keynote presentation) Paris, July (2002) 13-20.

42) CERMAV: 'Centre de recherches sur les molécules végétales' Unité propre CNRS ; BP 53 Grenoble Cedex 9 France. 